\title{
Glucagonoma-associated dilated cardiomyopathy refractory to somatostatin analogue therapy
}

\author{
Michal Barabas1,*, Isabel Huang-Doran2,*, Debbie Pitfield1, Hazel Philips, \\ Manoj Goonewardene ${ }^{3}$, Ruth T Casey ${ }^{1}$ and Benjamin G Challis ${ }^{1,4}$
}

${ }^{1}$ Wolfson Diabetes \& Endocrine Clinic, Cambridge University Hospitals NHS Foundation Trust, ${ }^{2}$ Wellcome-MRC Institute of Metabolic Science, Addenbrooke's Hospital, Cambridge, UK, 3Department of Cardiology, Bedford Hospital NHS Trust, Bedford, UK, and ${ }^{4}$ MED Biotech Unit, Clinical Discovery Unit, AstraZeneca, Cambridge, UK

*(M Barabas and I Huang-Doran contributed equally to this work)

Correspondence should be addressed to R T Casey or B G Challis Email

rc674@medschl.cam.ac.uk or bc340@medschl.cam.ac.uk

\section{Summary}

A 67-year-old woman presented with a generalised rash associated with weight loss and resting tachycardia. She had a recent diagnosis of diabetes mellitus. Biochemical evaluation revealed elevated levels of circulating glucagon and chromogranin B. Cross-sectional imaging demonstrated a pancreatic lesion and liver metastases, which were octreotideavid. Biopsy of the liver lesion confirmed a diagnosis of well-differentiated grade 2 pancreatic neuroendocrine tumour, consistent with metastatic glucagonoma. Serial echocardiography commenced 4 years before this diagnosis demonstrated a progressive left ventricular dilatation and dysfunction in the absence of ischaemia, suggestive of glucagonomaassociated dilated cardiomyopathy. Given the severity of the cardiac impairment, surgical management was considered inappropriate and somatostatin analogue therapy was initiated, affecting clinical and biochemical improvement. Serial cross-sectional imaging demonstrated stable disease 2 years after diagnosis. Left ventricular dysfunction persisted, however, despite somatostatin analogue therapy and optimal medical management of cardiac failure. In contrast to previous reports, the case we describe demonstrates that chronic hyperglucagonaemia may lead to irreversible left ventricular compromise. Management of glucagonoma therefore requires careful and serial evaluation of cardiac status.

\section{Learning points:}

- In rare cases, glucagonoma may present with cardiac failure as the dominant feature. Significant cardiac impairment may occur in the absence of other features of glucagonoma syndrome due to subclinical chronic hyperglucagonaemia.

- A diagnosis of glucagonoma should be considered in patients with non-ischaemic cardiomyopathy, particularly those with other features of glucagonoma syndrome.

- Cardiac impairment due to glucagonoma may not respond to somatostatin analogue therapy, even in the context of biochemical improvement.

- All patients with a new diagnosis of glucagonoma should be assessed clinically for evidence of cardiac failure and, if present, a baseline transthoracic echocardiogram should be performed. In the presence of cardiac impairment these patients should be managed by an experienced cardiologist. 


\section{Background}

We describe dilated cardiomyopathy as a rare clinical manifestation of the glucagonoma syndrome. In contrast with previous reports, cardiac function did not improve following initiation of somatostatin analogue therapy, despite biochemical resolution of hyperglucagonaemia. We recommend that all patients diagnosed with the glucagonoma syndrome undergo regular clinical examination for the presence of cardiac dysfunction, and if detected, get it thoroughly investigated and managed by an experienced cardiologist.

\section{Case presentation}

A 67-year-old female of Europid descent presented to the dermatology service with a 2-year history of a fleeting, generalised rash. The rash was erythematous with brown crusting, scaly and non-itchy, and particularly affected the legs, perineum and lower back (Fig. 1A). The appearances were in keeping with necrolytic migratory erythema, prompting referral to endocrinology.
On further inquiry, the patient reported profound weight loss, inappetence, reduced exercise tolerance, palpitations and peripheral oedema, all evolving over a 2-year period. There was no history of flushing, chest or abdominal pain, change in bowel habit or jaundice. A diagnosis of early diabetes mellitus had been made 2 years prior, which was well controlled with a single oral hypoglycaemic agent (40 mg gliclazide once daily). The patient reported pre-existing hypertension and osteoporosis. Notably, she had been investigated 4 years ago for left ventricular impairment of unknown aetiology. There was no history of venous thromboembolic disease. Regular medications included gliclazide, bisoprolol, alendronate, calcium and vitamin D. There was no family history of endocrinopathy (including multiple endocrine neoplasia), malignancy or premature cardiovascular disease. The patient consumed fewer than 10 units of alcohol per week and was a lifelong non-smoker.

On clinical examination the patient was slim with a BMI of $18.2 \mathrm{~kg} / \mathrm{m}^{2}$. She was tachycardic (resting heart rate ranging between 80 and 100 beats per minute) and hypotensive (blood pressure ranging from 80/40
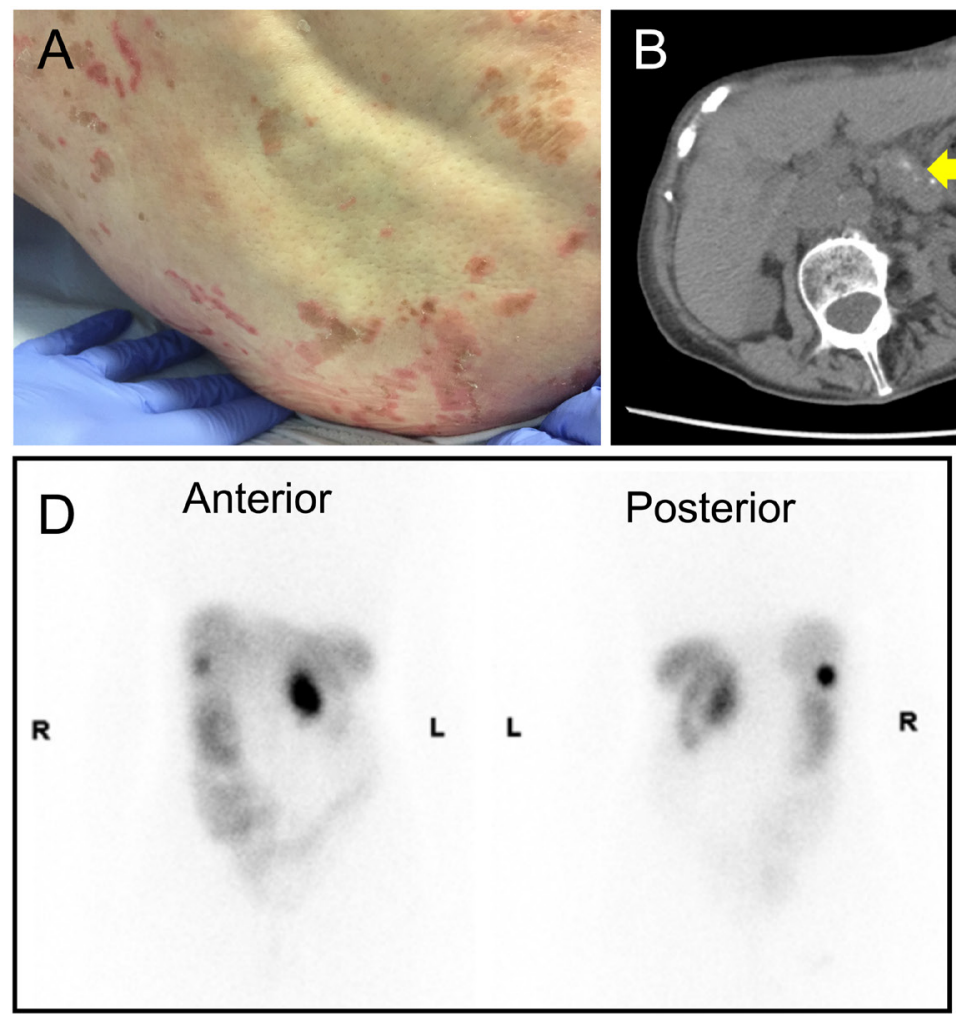
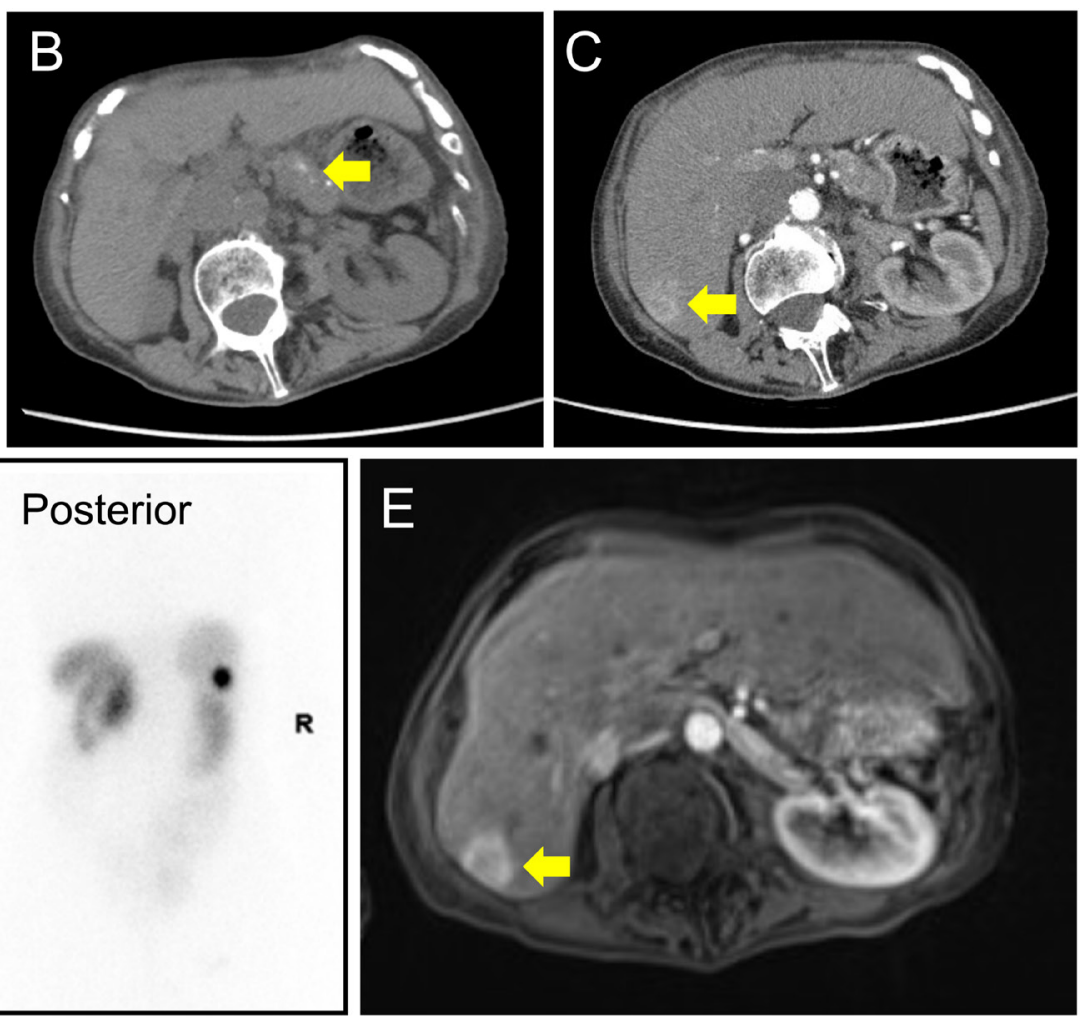

\section{Figure 1}

Clinical and radiological features at diagnosis. (A) Representative photograph of the patient's rash at presentation (gluteal region). (B and C) Crosssectional imaging at presentation, showing pancreatic (B) and hepatic (C) lesions. (D) Octreotide scintigraphy showing octreotide avidity in the pancreas and liver segment VI (anterior and posterior views). (E) Gadolinium-enhanced MRI images of the pancreatic and hepatic lesions. 
to $100 / 60 \mathrm{mmHg}$ ). Auscultation of the heart revealed normal S1 and S2 with no added sounds. Bilateral pitting oedema was appreciated in the lower limbs. Respiratory and abdominal examinations were unremarkable. Examination of the skin revealed an ongoing widespread blistering rash with areas of confluence covering the back and gluteal regions.

\section{Investigation}

Biochemical investigations revealed markedly elevated fasting plasma glucagon (>500 pmol/L; normal range: 0-50 pmol/L) and chromogranin B (526 pmol/L; normal range: 0-150 pmol/L) (Table 1). Haematological indices were unremarkable.

Cross-sectional imaging by computed tomography (CT) demonstrated a $51 \times 30 \mathrm{~mm}$ heterogeneous enhancing mass lesion in the body of the pancreas, as well as a $15 \mathrm{~mm}$ nodule in the head of the pancreas (Fig. 1B). Low attenuation lesions were seen in segments IV and VI of the liver (Fig. 1C), in addition to several enlarged mesenteric lymph nodes. Left ventricular dilatation, interlobular septal thickening and a left pleural effusion were noted.

Collectively, the clinical, biochemical and radiological findings were suggestive of a metastatic pancreatic glucagonoma. An octreotide scan demonstrated octreotide avidity in the pancreatic body and liver segment VI (Fig. 1D). Similarly, gadolinium-enhanced magnetic resonance imaging (MRI) of the liver demonstrated restricted diffusion in segment VI only, indicating that the other two liver lesions were benign cysts (Fig. 1E). Ultrasound-guided biopsy of the segment VI lesion confirmed a metastatic well-differentiated neuroendocrine tumour, with strong diffuse staining for synaptophysin, CD56 and CDX2; patchy but strong immunopositive staining for PAX8; and weak staining for chromogranin A. There was no immunopositive staining for glucagon or TTF-1. MIB-1 index was 2-5\%. The tumour was classified as a well-differentiated grade 2 pancreatic neuroendocrine tumour (NET) with liver metastases.

The abnormal cardiac appearances on CT prompted further evaluation. An electrocardiogram (ECG) demonstrated sinus tachycardia with left bundle branch block. Transthoracic echocardiography revealed severe left ventricular (LV) dysfunction and dilatation, with an ejection fraction of 20-25\%, and a left ventricular internal diameter in diastole (LVIDd) of $7.0 \mathrm{~cm}$ (normal range: $3.5-$ $5.6 \mathrm{~cm})$, consistent with dilated cardiomyopathy (DCM). Left atrial dilatation was also noted. Right ventricular size and function were normal. There was no evidence of significant valvular pathology (mild mitral regurgitation only). Indeed, echocardiography performed 4 years prior to diagnosis had similarly demonstrated severely reduced $\mathrm{LV}$ function with mild LV dilatation (LVIDd $5.5 \mathrm{~cm}$ ). Cardiac MRI subsequently confirmed a reduced LV ejection fraction with normal right heart function. There were no radiological features of myocardial oedema, scar or fibrosis. Gadolinium perfusion was normal, with no late gadolinium enhancement. Collectively these findings excluded ischaemic cardiomyopathy. There was no history of arrhythmia. There were no clinical or radiological features to suggest infiltrative cardiac disease or myocarditis. Of note, however, a viral screen was not undertaken at the time of initial diagnosis. There was no history of excessive alcohol consumption or use of cardiotoxic medications, nor was there a family history of cardiomyopathy. A diagnosis of glucagonoma-induced cardiomyopathy was therefore made.

\section{Treatment}

The patient's severely impaired LV function and general frailty were felt to be prohibitive for safe surgery, and a medical therapy with a somatostatin analogue was therefore recommended. Lanreotide (120 mg every 28 days) was initiated, with nutritional support provided

Table 1 Biochemical evaluation at baseline and during somatostatin analogue therapy.

\begin{tabular}{|c|c|c|c|c|}
\hline & \multirow[b]{2}{*}{ Reference range } & \multicolumn{3}{|c|}{ Duration of lanreotide treatment (months) } \\
\hline & & Baseline & 6 months & 24 months \\
\hline Glucagon (pmol/L) & $0-50$ & $>500$ & 67 & 100 \\
\hline Chromogranin A (pmol/L) & $0-60$ & 35 & 16 & 27 \\
\hline Chromogranin B (pmol/L) & $0-150$ & 526 & 308 & 368 \\
\hline Vasoactive intestinal peptide (pmol/L) & $0-30$ & 6 & 3 & 3 \\
\hline Somatostatin (pmol/L) & $0-150$ & 38 & 20 & 35 \\
\hline Gastrin (pmol/L) & $0-40$ & 28 & 8 & 35 \\
\hline Pancreatic polypeptide (pmol/L) & $0-300$ & 151 & 40 & 63 \\
\hline
\end{tabular}

All gut hormones were assayed in the fasting state. 
Table 2 Serial echocardiography at baseline and during somatostatin analogue therapy.

\begin{tabular}{l}
$\begin{array}{l}\text { Duration of } \\
\text { treatment (months) }\end{array}$ \\
\hline-46 \\
0 \\
6 \\
13 \\
21 \\
\hline
\end{tabular}

\begin{tabular}{cll}
\cline { 1 - 1 } LVIDd, $\mathbf{c m}$ & & \multicolumn{2}{l}{ LVEF, \% } \\
\cline { 1 - 1 } 5.5 & & $\begin{array}{c}\text { 'Severely reduced } \\
\text { systolic function' }\end{array}$ \\
7.0 & & $20-25$ \\
7.2 & & 28 \\
7.3 & & $20-25$ \\
6.9 & 28 \\
\hline
\end{tabular}

LVIDd, left ventricular internal diameter in diastole; LVEF, left ventricular ejection fraction.

by a specialist dietician. Cardiac function was optimised medically using a combination of beta-blockade, angiotensin converting enzyme inhibition (later switched to angiotensin II receptor blockade) and spironolactone.

\section{Outcome and follow-up}

Three months after initiation of lanreotide, the patient reported significant clinical improvement including resolution of the rash, improved appetite, weight gain and minimal peripheral oedema. Her exercise tolerance was stable and there was no subjective dyspnoea. Repeat biochemical investigation after 6 months of lanreotide therapy demonstrated marked improvement in both fasting glucagon $(67 \mathrm{pmol} / \mathrm{L})$ and chromogranin B (308 pmol/L) concentrations (Table 1). Cross-sectional imaging showed stable appearances of the primary pancreatic tumour, hepatic metastases and mesenteric lymph nodes; however, there was persistent dilatation of the left ventricle with worsening pulmonary congestion. Consistent with this, repeat echocardiography demonstrated an unchanged LVEF and LVIDd, indicating ongoing severe LV impairment (Table 2).

Eleven months after commencement of lanreotide treatment, the patient presented to her local hospital with worsening lethargy, tachycardia and clinical features suggestive of acute decompensation of cardiac failure. Cross-sectional imaging demonstrated new moderate bilateral pleural effusions, cardiomegaly with severe tricuspid regurgitation and progressive pulmonary congestion. Echocardiography demonstrated an LVEF of $20-25 \%$ and LVIDd of $7.3 \mathrm{~cm}$, indicating ongoing severe LV failure. There was no evidence of an acute ischaemic event. Following further medical optimisation (furosemide $40 \mathrm{mg}$, spironolactone $25 \mathrm{mg}$, bisoprolol $5 \mathrm{mg}$, ramipril $2.5 \mathrm{mg}$ and losartan $50 \mathrm{mg}$ ), her symptoms returned to their baseline level and

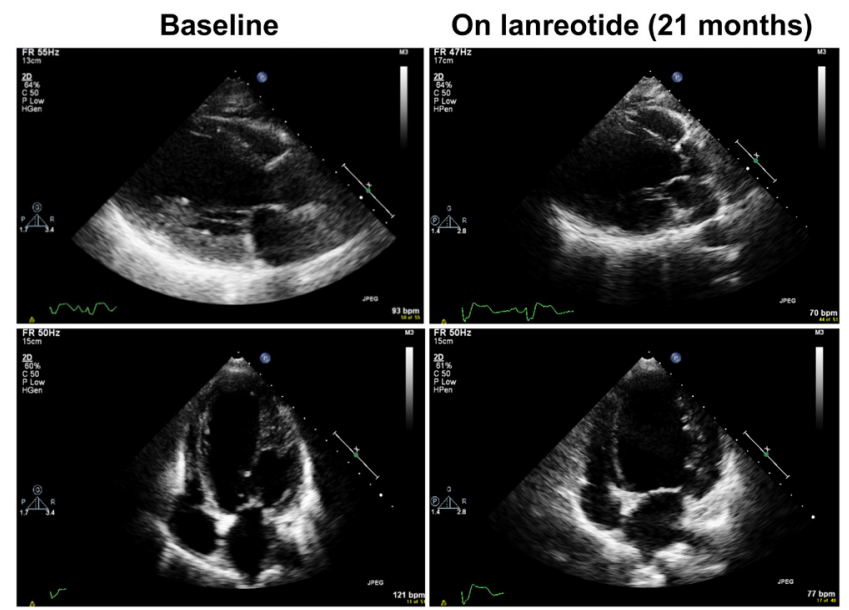

Figure 2

Echocardiographic features of dilated cardiomyopathy before and after somatostatin analogue therapy. Representative four-chamber views showing left ventricular dilatation at baseline (left) and after 21 months of lanreotide therapy (right).

she reported good exercise tolerance. Treatment with lanreotide continued.

At her latest clinical review, 24 months after commencement of lanreotide, the patient reported ongoing symptomatic control and fasting gut-hormone levels were stable (Table 1). Surveillance imaging by contrast enhanced CT demonstrated stable pancreatic disease, however repeat echocardiography continued to show a severely dilated left ventricle with severe $\mathrm{LV}$ dysfunction despite optimisation of medical therapy (Fig. 2). NT pro-BNP was markedly elevated at $15189 \mathrm{pg} / \mathrm{mL}$. Biventricular pacemaker insertion is planned.

\section{Discussion}

Glucagonomas are rare tumours with an annual incidence of 1 per 20-40 million population (1). $80 \%$ are sporadic, with the remainder associated with inherited tumour predisposition syndromes such as multiple endocrine neoplasia type 1 (MEN-1). Patients may be asymptomatic or present with a range of features typical of the glucagonoma syndrome, including necrolytic migratory erythema, weight loss, glucose intolerance, painful glossitis, stomatitis, diarrhoea, thromboembolic disease and psychiatric illness (1). Less commonly recognised are paraneoplastic phenomena associated with impaired processing of the proglucagon prohormone and aberrant tumoural secretion of proglucagon-derived peptides, including GLP-1 and GLP-2, which may induce hyperinsulinaemic hypoglycaemia and gastrointestinal dysfunction, respectively $(1,2)$. In addition, in three 
Table 3 Biochemical and cardiac response to treatment of dilated cardiomyopathy associated with glucagonoma.

\begin{tabular}{|c|c|c|c|c|c|c|c|c|}
\hline \multirow[b]{2}{*}{ Reference } & \multirow[b]{2}{*}{ Age (year) } & \multirow[b]{2}{*}{ Sex } & \multirow[b]{2}{*}{ Treatment } & \multicolumn{3}{|c|}{ Glucagon levels } & \multicolumn{2}{|c|}{ LVEF, \% } \\
\hline & & & & Baseline & Post treatment & Reference range & Baseline & Post treatment \\
\hline (4) & 54 & $\mathrm{~F}$ & Pancreatectomy & $1261 \mathrm{pg} / \mathrm{mL}$ & $26 \mathrm{pg} / \mathrm{mL}$ & $20-100 \mathrm{pg} / \mathrm{mL}$ & 15 & 55 \\
\hline (5) & 51 & $\mathrm{~F}$ & Octreotide infusion & ND & ND & & 10 & 45 \\
\hline (6) & 64 & M & $\begin{array}{l}\text { Distal } \\
\text { pancreatectomy }\end{array}$ & $97 \mathrm{pmol} / \mathrm{L}$ & Normal & $<50 \mathrm{pmol} / \mathrm{L}$ & 30 & 67 \\
\hline This case & 67 & $\mathrm{~F}$ & Lanreotide & $>500 \mathrm{pmol} / \mathrm{L}$ & $67 \mathrm{pmol} / \mathrm{L}$ & $<50 \mathrm{pmol} / \mathrm{L}$ & $20-25$ & 28 \\
\hline
\end{tabular}

LVEF, left ventricular ejection fraction; ND, not done.

previously published reports, decompensated heart failure and dilated cardiomyopathy were posited as rare clinical manifestations of the glucagonoma syndrome $(3,4,5$; Table 3). Herein we describe the fourth reported case of severe cardiac dysfunction in a patient with glucagonoma.

In 2004, Chang-Chretien and colleagues described the first case of acute congestive heart failure secondary to dilated cardiomyopathy as the presenting feature of glucagonoma in a 54-year-old female (3). In their report, biochemical investigations identified markedly elevated serum glucagon levels (12 times the upper limit of normal) in association with necrolytic migratory erythema, dilated cardiomyopathy, severely impaired left ventricular function (LVEF 15\%) and resting tachycardia. Surgical resection of the tumour resulted in complete biochemical resolution and, strikingly, reversal of the dilated cardiomyopathy (normal LV end-diastolic dimensions and LVEF 55\%) and normalisation of the heart rate. Subsequently, Demir et al. (4) also reported complete resolution of dilated cardiomyopathy 1 month following surgical resection of a pancreatic glucagonoma. Zhang et al. (5) described severe left ventricular dysfunction (LV ejection fraction of 10\%) presenting with cardiogenic shock in a 51-year-old female with MEN-1, and a histologically confirmed metastatic glucagonoma. Cardiac biopsy demonstrated cardiomyocyte hypertrophy. Additional investigations failed to identify an alternative explanation for the severely impaired left ventricle and a diagnosis of glucagon-induced acute heart failure was made. Interestingly, a continuous intravenous infusion of octreotide resulted in an immediate improvement of LV function and following 3 weeks of treatment LVEF had improved to $45 \%$.

In our patient, dilated cardiomyopathy was diagnosed 4 years prior to the discovery of the glucagonoma, with the finding of cardiomegaly on cross-sectional imaging prompting cardiac re-evaluation. In the absence of an alternative aetiology, a diagnosis of glucagon-induced cardiomyopathy was made. Due to both severe cardiac dysfunction and metastatic disease the possibility of surgical resection was not pursued. Alternatively, we opted to treat our patient medically with somatostatin analogues anticipating, based on previous clinical reports, that, in addition to effects on tumour growth and hormone production, this treatment would prove beneficial for the LVEF. In contrast to previous reports, however, and despite biochemical improvement and tumour response to lanreotide, there was no improvement in LV function or dimensions. It remains possible that an alternative aetiology caused the development of dilated cardiomyopathy in our patient, thereby accounting for the cardiac resistance to somatostatin analogues. Alternatively, the prolonged exposure to supra-physiological glucagon levels may have induced irreversible pathological effects on the myocardium that cannot be overcome with medical treatment.

In addition to well-established actions of glucagon on glucose homeostasis, evidence from several preclinical and clinical studies suggest that glucagon has direct actions on the cardiovascular system (6). Indeed, glucagon is a recognised treatment for beta-adrenoceptor antagonist overdose owing to its positive inotropic and chronotropic properties. Supra-physiological levels of glucagon may account for resting tachycardia observed in some patients with glucagonoma.

Glucagon mediates its effects through binding and activation of the glucagon receptor (GCGR), a G-proteincoupled receptor that is expressed in a variety of tissues in animals and humans. In cardiac ventricles, however, marked interspecies differences exist with regard to GCGR expression. For example, functional GCGR is expressed in the left ventricle of mice and rats, but not in the left ventricle of humans $(7,8)$. In contrast, abundant glucagon-like peptide 1 receptor (GLP-1R) mRNA is expressed in all four chambers of the human heart, whereas glucagon-like peptide 2 receptor (GLP-2R) mRNA is expressed to a much lesser extent (7). The variability in GCGR expression in the human heart, along with cross reactivity with other related G-protein-coupled receptors, may explain the apparently preferential effect of supra- 
physiological hyperglucagonaemia, and/or aberrantly processed proglucagon-derived peptides, on the left ventricle $(4,5,9)$. These observations are directly relevant to ongoing efforts to characterise the effects of glucagon and related peptides on human cardiac physiology (6).

In summary, we have reported the fourth clinical case of dilated cardiomyopathy and LV dysfunction in association with a metastatic glucagonoma. In contrast to previous reports, we observed no improvement in cardiac function following improvement in glucagon levels with somatostatin analogue treatment. We propose that dilated cardiomyopathy be considered a rare clinical manifestation of the glucagonoma syndrome. Accordingly, we recommend that individuals with glucagonoma undergo cardiac assessment at the time of diagnosis to ensure that appropriate clinical management and follow-up is initiated.

\section{Declaration of interest}

B G C is an employee of AstraZeneca. The other authors have nothing to disclose.

\section{Funding}

This research did not receive any specific grant from any funding agency in the public, commercial or not-for-profit sector

\section{Patient consent}

Written informed consent for publication of clinical details was obtained from the patient.

\section{Author contribution statement}

$\mathrm{M} \mathrm{B}$ and I H D performed the literature review and wrote the manuscript. $D \mathrm{P}, \mathrm{H} \mathrm{P}$, and $\mathrm{M} \mathrm{G}$ contributed to the clinical care of the patient, and reviewed the manuscript. R T C and B C G are the patient's responsible physicians and wrote the manuscript.

\section{References}

1 Jensen RT, Cadiot G, Brandi ML, de Herder WW, Kaltsas G, Komminoth P, Scoazec JY, Salazar R, Sauvanet A, Kianmanesh R, et al. ENETS Consensus Guidelines for the management of patients with digestive neuroendocrine neoplasms: functional pancreatic endocrine tumor syndromes. Neuroendocrinology 201295 98-119. (https://doi.org/10.1159/000335591)

2 Wewer Albrechtsen NJ, Kuhre RE, Pedersen J, Knop FK \& Holst JJ. The biology of glucagon and the consequences of hyperglucagonemia. Biomarkers in Medicine 201610 1141-1151. (https://doi.org/10.2217/bmm-2016-0090)

3 Challis BG, Albrechtsen NJ, Bansiya V, Burling K, Barker P, Hartmann B, Gribble F, O'Rahilly S, Holst JJ \& Simpson HL. Heterogeneity of glucagonomas due to differential processing of proglucagon-derived peptides. Endocrinology, Diabetes and Metabolism Case Reports 20152015 150105. (https://doi.org/10.1530/EDM-150105)

4 Chang-Chretien K, Chew JT \& Judge DP. Reversible dilated cardiomyopathy associated with glucagonoma. Heart 200490 e44. (https://doi.org/10.1136/hrt.2004.036905)

5 Demir OM, Paschou SA, Ellis HC, Fitzpatrick M, Kalogeropoulos AS, Davies A, Thompson J, Davies SW \& Grapsa J. Reversal of dilated cardiomyopathy after glucagonoma excision. Hormones $2015 \mathbf{1 4}$ 172-173. (https://doi.org/10.14310/horm.2002.1557)

6 Petersen KM, Bogevig S, Holst JJ, Knop FK \& Christensen MB. Hemodynamic effects of glucagon: a literature review. Journal of Clinical Endocrinology and Metabolism 2018103 1804-1812. (https:// doi.org/10.1210/jc.2018-00050)

7 Baggio LL, Yusta B, Mulvihill EE, Cao X, Streutker CJ, Butany J, Cappola TP, Margulies KB \& Drucker DJ. GLP-1 receptor expression Within the human heart. Endocrinology 2018159 1570-1584. (https://doi.org/10.1210/en.2018-00004)

8 Ali S, Ussher JR, Baggio LL, Kabir MG, Charron MJ, Ilkayeva O, Newgard CB \& Drucker DJ. Cardiomyocyte glucagon receptor signaling modulates outcomes in mice with experimental myocardial infarction. Molecular Metabolism 20154 132-143. (https://doi. org/10.1016/j.molmet.2014.11.005)

9 Zhang K, Lehner LJ, Praeger D, Baumann G, Knebel F, Quinkler M\& Roepke TK. Glucagonoma-induced acute heart failure. Endocrinology, Diabetes and Metabolism Case Reports 20142014 140061. (https://doi. org/10.1530/EDM-14-0061)

Received in final form 17 January 2019

Accepted 11 February 2019 